\title{
INITIAL UNSTEADY FREE CONVECTIVE FLOW PAST AN INFINITE VERTICAL PLATE WITH RADIATION AND MASS TRANSFER EFFECTS
}

\author{
A. KHAN* and R. SOLANKI \\ Department of Mathematics and Statistics \\ Jai Narain Vyas University, Jodhpur, INDIA \\ E-mails: draiyubkhan@gmail.com; rahul.27.solanki@gmail.com
}

\begin{abstract}
An exact solution and analysis of an initial unsteady two dimensional free convection flow, heat and mass transfer in the presence of thermal radiation along an infinite fixed vertical plate when the plate temperature is instantaneously raised, is presented. The fluid considered is a gray, absorbing emitting radiation but a nonscattering medium. Three cases have been discussed, in particular, namely, (i) when, the plate temperature is instantaneously raised to a higher constant value, (ii) when, the plate temperature varies linearly with time and (iii) when, the plate temperature varies non-linearly with time. A close form general solution for all the cases has been obtained in terms of repeated integrals of error functions. In two particular cases, the solutions in terms of the repeated integrals of error functions have been further simplified to forms containing only error functions. It is observed that for an increase in the radiation parameter $N$ or a decrease in the Grashof number Gr or Gm, there is a fall in the velocity or temperature, but compared to the no radiation case or no diffusing species, there is a rise in the velocity and temperature of the fluid.
\end{abstract}

Key words: free convection flow, porous medium, heat transfer, mass transfer, MHD.

\section{Introduction}

The phenomenon of free convection arises in a fluid when temperature changes cause density variations leading to buoyancy forces acting on the fluid elements. This process of heat transfer is encountered in aeronautics, fluid fuel nuclear reactor and chemical engineering. Soundalgekar [1] presented an exact solution for the flow past an infinite vertical isothermal plate impulsively started in a viscous incompressible fluid and Patil and Soundalgekar [2] considered the same problem and presented an exact solution for an accelerated vertical infinite plate. Flow past a vertical oscillating plate with variable temperature has been discussed by Vighnesham et al. [3].

Korycki [4] stated that in textiles (i.e., industrial textiles, textiles designed for use under hermetic protective barrier, multilayer clothing materials, etc.) and in textile structures (i.e., needle heating in heavy industrial sewing), radiative heat transfer problems are encountered as well. Evidently, high temperature phenomena cannot be ignored. Therefore, it is more realistic to study the problem of an unsteady free convection flow with radiative heat transfer past a vertical porous plate. Chaudhary and Jain [5] studied the problem of an unsteady free convective flow past an impulsively started vertical surface, taking into account the effects of radiative heat transfer and suction. Heat and mass transfer effects on a moving plate in the presence of thermal radiation were studied by Muthukumarswamy et al. [6] using the Laplace technique.

Chen [7] studied the problem of combined heat and mass transfer of an electrically conducting fluid in MHD natural convection, adjacent to a vertical surface with Ohmic heating. Chandran et al. [8] presented natural convection with ramped wall temperature. Several authors, namely, Muthucumaraswamy [9], Pathak et al. [10], Chandrakala [11] and Deka and Das [12], studied the problems of free convection flows, modeled

\footnotetext{
${ }^{*}$ To whom correspondence should be addressed
} 
under the assumptions of constant surface temperature, ramped wall temperature, or constant surface heat flux.

However, in practical problems when the plate temperature is raised, it may, initially increase linearly or non-linearly with time and therefore it is interesting to know how such initial thermal conditions of the plate affect the skin-friction, flow and heat transfer. Parihar [13] studied cases of such initial thermal conditions for a free convective flow in the absence of thermal radiation and species concentration. Furthermore, the free convection flows together with heat and mass transfer are of great importance in geophysics, aeronautics, and engineering. In several processes such as drying, evaporation of water at body surface, energy transfer in a wet cooling tower, and flow in a desert cooler, heat and mass transfer occur simultaneously. In view of such applications, several authors, namely, Chandrakala et al. [14], Muthucumaraswamy et al. [15], Muthucumaraswamy et al. [16], Prasad et al. [17], Narahari et al. [18], Rajesh [19], Brewster [20] and Abramowitz [21] investigated free convection flows with simultaneous heat and mass transfer phenomenon. However, the exact solutions to these problems have been obtained by few authors, namely Mebine et al. [22], Narahari et al. [23], Narahari et al. [18] and Rajesh [19].

In the present paper, an exact solution to the problem of the initial unsteady free convective flow and heat transfer along an infinite vertical plate, taking the thermal radiation and species concentration into account, has been obtained in three cases, namely, (i) when the plate temperature is instantaneously raised to a higher constant value, (ii) when the plate temperature varies linearly with time and (iii) when the plate temperature varies non-linearly with time.

The results are discussed in detail in section 5 .

\section{Formulation of the problem}

Consider an initial unsteady two-dimensional free convective flow of viscous incompressible radiating fluid, with species concentration past an infinite vertical plate fixed in infinite mass of fluid which is otherwise at rest. At time $t \leq 0$, the temperature of the plate and fluid is $T_{\infty}$ and for $t>0$, the plate temperature is instantaneously raised to $T_{w}(t)$. It is assumed that the temperature difference between the plate and the fluid is small, so that the fluid properties may be taken as constant except for the influence of density variation in the body force term. The $x$-axis is taken along the plate in vertical upward direction and the $y$-axis is normal to it. Then, under the usual Boussinesq's approximations, the equations governing the flow, neglecting frictional heat, are given by

$$
\begin{aligned}
& \frac{\partial u}{\partial t}=v \frac{\partial^{2} u}{\partial y^{2}}+g \beta\left(T-T_{\infty}\right)+g \beta^{*}\left(C-C_{\infty}\right), \\
& \frac{\partial T}{\partial t}=\frac{\kappa}{\rho C p} \frac{\partial^{2} T}{\partial y^{2}}-\frac{1}{\rho C p} \frac{\partial q_{r}}{\partial y} \\
& \frac{\partial C}{\partial t}=D \frac{\partial^{2} C}{\partial y^{2}}
\end{aligned}
$$

with the following initial and boundary conditions

$$
\begin{array}{ll}
t<0: \quad u=0, \quad T=T_{\infty}, \quad C=C_{\infty} \quad \text { for all } y, \\
t \geq 0: \quad u=0, \quad T=T_{w}(t), \quad C=C_{w}(t) \quad \text { at } \quad y=0, \\
u=0, \quad T=T_{\infty}, \quad C=C_{\infty} \quad \text { as } y \rightarrow \infty .
\end{array}
$$


Here $u$ is the velocity component in the $x$ direction, $t$ is the time, $v$ is the kinematic viscosity, $g$ is the acceleration due to gravity, $\beta$ is the coefficient of volume expansion. $\beta^{*}$ is the volumetric coefficient of expansion with species concentration, $T$ is the temperature of the fluid, $T_{\infty}$ is the temperature of the fluid far away from the plate. $T_{w}$ is the plate temperature, $\kappa$ is the thermal conductivity of the fluid, $\rho$ is the density, $C_{p}$ is the specific heat at constant pressure, $q_{r}$ is the radiative heat flux in the y direction, $C$ is the species concentration, $C_{\infty}$ is the concentration at infinity and $D$ is the chemical molecular diffusivity.

The temperature of the plate and species concentration are now assumed to have the following form

$$
\begin{aligned}
& T_{w}(t)=T_{\infty}\left(1+A t^{r-1}\right), \\
& C_{w}(t)=C_{\infty}\left(1+A t^{r-1}\right)
\end{aligned}
$$

where, $A=\left(u_{0}^{2} / v\right)^{r-1}, u_{0}$ is a constant with dimension of velocity and $r \geq 1$ is an integer. It may be noted that $r=1$ is the case of an isothermal plate (when the plate temperature is instantaneously raised to a constant higher temperature), $r=2$ is the case of plate temperature varying linearly with time and $r \geq 3$ is the case of plate temperature varying non-linearly with time. These three particular cases have been discussed in particular.

We assume the Rosseland approximation [3], for the radiative heat flux, which leads to

$$
q_{r}=-\frac{4 \sigma}{3 \kappa^{*}} \frac{\partial T^{4}}{\partial y}
$$

where $\sigma$ is the Stefan-Boltzmann constant and $\kappa^{*}$ is the mean absorption coefficient.

If the temperature differences within the flow are sufficiently small such that $T^{4}$ may be expressed as a linear function of the temperature, then the Taylor series for $T^{4}$ about $T_{\infty}$, after neglecting higher order terms, is given by

$$
T^{4}=4 T_{\infty}^{3} T-3 T_{\infty}^{4} .
$$

In view of Eqs (2.7) and (2.8), Eq.(2.2) reduces to

$$
\frac{\partial T}{\partial t}=\frac{\kappa}{\rho c_{p}} \frac{\partial^{2} T}{\partial y^{2}}+\frac{16 \sigma T_{\infty}^{3}}{3 \rho c_{p} \kappa^{*}} \frac{\partial^{2} T}{\partial y^{2}} .
$$

Let us introduce the following non-dimensional quantities

$$
\begin{aligned}
& y^{*}=\frac{y u_{0}}{v}, \quad u^{*}=\frac{u}{u_{0}}, \quad t^{*}=\frac{t u_{0}^{2}}{v}, \quad \theta=\frac{T-T_{\infty}}{T_{w}-T_{\infty}}, \\
& \operatorname{Pr}=\frac{\mu c_{p}}{\kappa}, \quad \mathrm{Gr}=\frac{v g \beta T_{\infty}}{u_{0}^{3}}, \quad N=\frac{\kappa^{*} k}{4 \sigma T_{\infty}^{3}}, \quad \mathrm{Sc}=\frac{v}{D}, \\
& C^{*}=\frac{C-C_{\infty}}{C_{w}-C_{\infty}}, \quad \mathrm{Gm}=\frac{v g \beta^{*} C_{\infty}}{u_{0}^{3}}, \quad u_{0}=\left(\frac{v}{t}\right)^{1 / 2}
\end{aligned}
$$


where Pr is the Prandtl number, Gr the Grashof number, Gm the Grashof number for mass transfer, Sc the Schmidt number, $N$ the radiation parameter and $u_{0}$ is a constant with dimension of velocity, in Eqs (2.1), (2.3), (2.4) and (2.9) which reduce to the following after dropping the astrisk sign

$$
\begin{aligned}
& \frac{\partial u}{\partial t}=\frac{\partial^{2} u}{\partial y^{2}}+\mathrm{Gr} \cdot t^{r-1} \theta+\mathrm{Gm} \cdot t^{r-1} C \\
& \frac{\partial \theta}{\partial t}+\frac{\theta(r-1)}{t}=\frac{1}{\operatorname{Pr}}\left(\frac{3 N+4}{3 N}\right) \frac{\partial^{2} \theta}{\partial y^{2}}, \\
& \frac{\partial C}{\partial t}+\frac{C(r-1)}{t}=\frac{1}{\mathrm{Sc}} \frac{\partial^{2} C}{\partial y^{2}},
\end{aligned}
$$

with the following initial and boundary conditions

$$
\begin{aligned}
& t<0: \quad u=0, \quad \theta=0, \quad C=0 \text { for all } y, \\
& t \geq 0: \quad u=0, \quad \theta=1, \quad C=1 \quad \text { at } \quad y=0, \\
& u=0, \quad \theta=0, \quad C=0 \quad \text { as } \quad y \rightarrow \infty .
\end{aligned}
$$

The partial differential Eqs (2.10), (2.11) and (2.12) with initial and boundary conditions (2.13) can be solved either by the Laplace-transform technique or by using similarity transformation.

\section{Solution of the problem}

We choose the latter technique and introduce the following similarity transformations

$$
\begin{aligned}
& u(y, t)=t^{r} f(\eta), \\
& \theta(y, t)=\varphi(\eta), \\
& C(y, t)=\psi(\eta), \\
& \text { and } \eta=\frac{y}{2 \sqrt{t}},
\end{aligned}
$$

into Eqs (2.10), (2.11) and (2.12) which reduce to the following ordinary differential equations

$$
\begin{aligned}
& f^{\prime \prime}+2 \eta f^{\prime}-4 r f+4 \mathrm{Gr} . \phi+\mathrm{Gm} \cdot \psi=0, \\
& \frac{1}{\operatorname{Pr} . \alpha} \phi^{\prime \prime}+2 \eta \phi^{\prime}-4(r-1) \phi=0, \\
& \frac{1}{\mathrm{Sc}} \psi^{\prime \prime}+2 \eta \psi^{\prime}-4(r-1) \psi=0,
\end{aligned}
$$


with the following boundary conditions

$$
\begin{aligned}
& \eta=0: \quad f=0, \quad \phi=1, \quad \psi=1, \\
& \eta \rightarrow \infty: \quad f=0, \quad \phi=0, \quad \psi=0 .
\end{aligned}
$$

Here, $\alpha=\frac{3 N}{3 N+4}$, and a prime denotes differentiation with respect to $\eta$.

The solutions of Eqs (3.2), (3.3) and (3.4) can be easily obtained in terms of repeated integrals of complementary error functions (see, e.g. Abramovitz and Stegun [21]). Equation (3.3) and (3.4), being independent of (3.2), is first solved, satisfying the boundary conditions (3.5). Then a particular integral of Eq.(3.2), in view of the solution of Eqs (3.3) and (3.4), is obtained by the method of undetermined coefficients (Bansal [24]) and finally, the complete solution of Eq.(3.2), satisfying the boundary conditions (3.5), is obtained. These solutions are as follows

$$
\begin{aligned}
& \theta=4^{r-1} \Gamma r i^{(2 r-2)} \operatorname{erf}_{\mathrm{c}}(\eta \sqrt{\operatorname{Pr} . \alpha}), \\
& C=4^{r-1} \Gamma r i^{(2 r-2)} \operatorname{erf}_{\mathrm{c}}(\eta \sqrt{\mathrm{Sc}}), \\
& u=\frac{t^{r} 4^{r} \Gamma r \mathrm{Gr}}{(1-\operatorname{Pr} . \alpha)}\left[i^{2 r} \operatorname{erf}_{\mathrm{c}}(\eta \sqrt{\operatorname{Pr} . \alpha})-i^{2 r} \operatorname{erf}_{\mathrm{c}}(\eta)\right]+ \\
& +\frac{t^{r} 4^{r} \Gamma r \mathrm{Gm}}{(1-\mathrm{Sc})}\left[i^{2 r} \operatorname{erf}_{\mathrm{c}}(\eta \sqrt{\mathrm{Sc}})-i^{2 r} \operatorname{erf}_{\mathrm{c}}(\eta)\right] .
\end{aligned}
$$

Here, the function $i^{n} \operatorname{erf}_{\mathrm{c}}(z)$ is the repeated integral of the complementary error function and is given by

$$
i^{n} \operatorname{erf}_{\mathrm{c}}(z)=\frac{2}{\sqrt{\pi}} \int_{z}^{\infty} \frac{(t-z)^{n}}{n !} e^{-t^{2}} d t, \quad n=0,1,2, \ldots
$$

The solution (3.8) is valid for $\operatorname{Pr} . \alpha \neq 1$ and $\mathrm{Sc} \neq 1$. In the case of $\operatorname{Pr} . \alpha=1$ or $\mathrm{Sc}=1$, on taking the limiting values, one can easily obtain the following expressions for the velocity.

Case (i): When Pr. $\alpha=1$ and $\mathrm{Sc} \neq 1$

$$
\begin{aligned}
& u=t^{r} 4^{r-1} \Gamma r \operatorname{Gr}\left[i^{2 r-2} \operatorname{erf}_{\mathrm{c}}(\eta)-4 r \cdot i^{2 r} \operatorname{erf}_{\mathrm{c}}(\eta)\right]+ \\
& +\frac{t^{r} 4^{r} \Gamma r \mathrm{Gm}}{(1-\mathrm{Sc})}\left[i^{2 r} \operatorname{erf}_{\mathrm{c}}(\eta \sqrt{\mathrm{Sc}})-i^{2 r} \operatorname{erf}_{\mathrm{c}}(\eta)\right] .
\end{aligned}
$$

Case (ii): When $\operatorname{Pr} . \alpha \neq 1$ and $\mathrm{Sc}=1$

$$
\begin{aligned}
& u=\frac{t^{r} 4^{r} \Gamma r \mathrm{Gr}}{(1-\operatorname{Pr} . \alpha)}\left[i^{2 r} \operatorname{erf}_{\mathrm{c}}(\eta \sqrt{\operatorname{Pr} . \alpha})-i^{2 r} \operatorname{erf}_{\mathrm{c}}(\eta)\right]+ \\
& +t^{r} 4^{r-1} \operatorname{\Gamma r} \mathrm{Gm}\left[i^{2 r-2} \operatorname{erf}_{\mathrm{c}}(\eta)-4 r \cdot i^{2 r} \operatorname{erf}_{\mathrm{c}}(\eta)\right] .
\end{aligned}
$$


Case (iii): When $\operatorname{Pr} . \alpha=1$ and $\mathrm{Sc}=1$

$$
u=t^{r} 4^{r-1} \Gamma r(\mathrm{Gr}+\mathrm{Gm})\left[i^{2 r-2} \operatorname{erf}_{\mathrm{c}}(\eta)-4 r \cdot i^{2 r} \operatorname{erf}_{\mathrm{c}}(\eta)\right]
$$

It may be noted that $\mathrm{Gm}=0$ is the case of absence of species concentration and $N \rightarrow \infty$ or $\alpha \rightarrow 1$ is the case of absence of the radiative heat flux. In the case of absence of both the species concentration and radiative heat flux, the above expressions for velocity reduce to those obtained by Tak and Parihar [12].

\section{Skin-friction coefficient}

The non-dimensional skin-friction at the plate is determined by the formula.

$$
C_{f}=\frac{\mu\left(\frac{\partial u}{\partial y}\right)_{y=0}}{\frac{1}{2} \rho u_{0}^{2}}=2 t^{r-\frac{1}{2}} \frac{\Gamma r}{\Gamma(r+1 / 2)}\left[\frac{\mathrm{Gr}}{(1+\sqrt{\operatorname{Pr} \alpha})}+\frac{\mathrm{Gm}}{(1+\sqrt{\mathrm{Sc}})}\right] .
$$

In the case of no radiation $(\alpha=1)$ and no mass transfer $(\mathrm{Gm}=0)$, the expression for $C_{f}$ becomes

$$
C_{f}=2 t^{r-\frac{1}{2}} \frac{\Gamma r}{\Gamma(r+1 / 2)}\left[\frac{\mathrm{Gr}}{(1+\sqrt{\operatorname{Pr} \alpha})}\right]
$$

\section{Results and discussion}

It may be noted from the non-dimensional Eqs (3.2) to (3.5) that the present problem is described by six parameters, namely Gr, Gm, Pr, Sc, $r$ and $\alpha($ or $N$ ). Since the effects of variation of $\mathrm{Pr}$ and $\mathrm{Sc}$ on the flow are well known, all numerical calculations have been performed for $\operatorname{Pr}=0.71$ and $\mathrm{Sc}=0.6$. It may be noted that $\alpha=1$ (or $N \rightarrow \infty$ ) is the case when the thermal radiation is neglected and Gm $=0$ is the case of absence of species concentration. The value of the parameter $r$ determines whether the temperature and concentration at the plate are constant or vary with time (see, Eqs (2.5) and (2.6)). We have chosen $r=1,2$ and 3 which describes three different physical situations of the flow and discussed the results as follows:

\section{Case (i) $r=1$ (Isothermal and isosolutal plate):}

This corresponds to the situation of an initial unsteady free convective flow when the temperature and concentration of the plate are raised to attain higher constant values instantaneously. In this case, we have drawn three-dimensional graphs for velocity distribution $u(y, t)$ for different values of $\mathrm{Gr}$, $\mathrm{Gm}$ and $N$, in Figs 1-4. Figure 1 depicts the developing velocity profiles in the presence of thermal radiation and diffusing species when $\mathrm{Gr}=4, \mathrm{Gm}=4$ and $N=3$, whereas Fig. 2 depicts the case when thermal radiation is neglected $(\alpha=1$ or $N \rightarrow \infty)$, Fig. 3 depicts the case when species concentration is absent $(\mathrm{Gm}=0)$ and Fig.4, when both radiation and concentration are absent $(\alpha=1$ and $\mathrm{Gm}=0)$. 


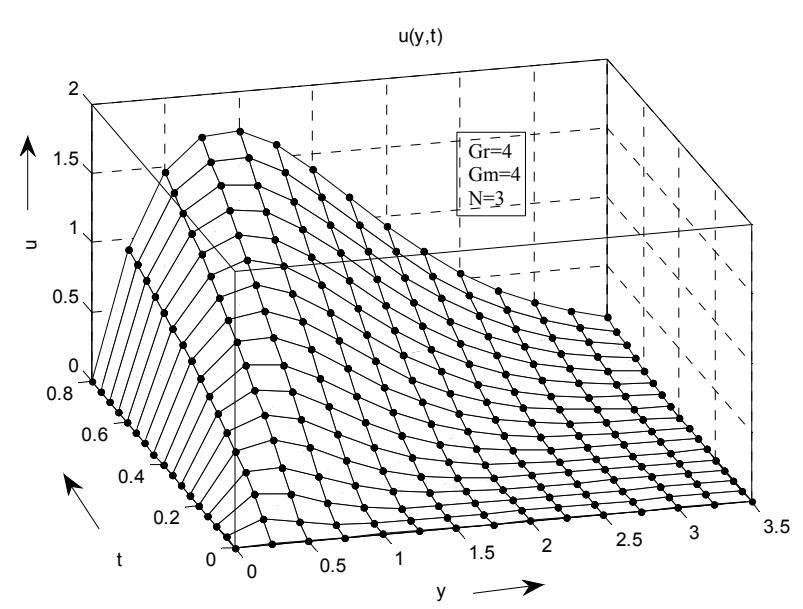

Fig.1. Variation of velocity with $y$ and $t$ in the case of isothermal plate $(r=1)$.

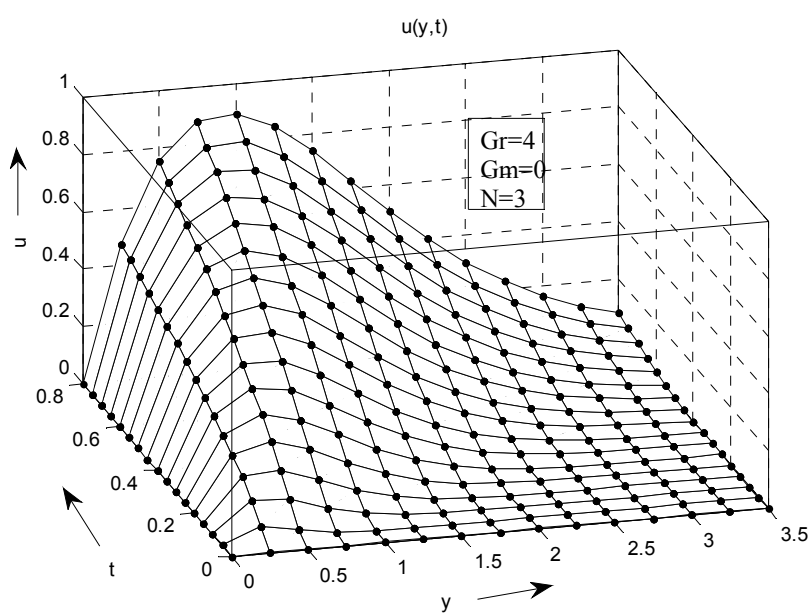

Fig.3. Variation of velocity with $y$ and $t$ in the case of isothermal plate $(r=1)$ in the absence of mass transfer.

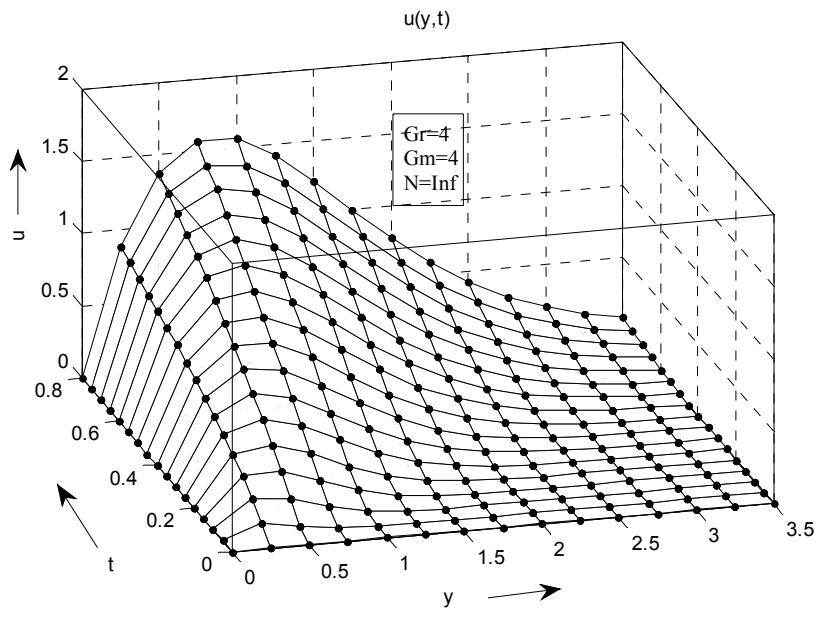

Fig.2. Variation of velocity with $y$ and $t$ in the case of isothermal plate $(r=1)$ in the absence of radiation.

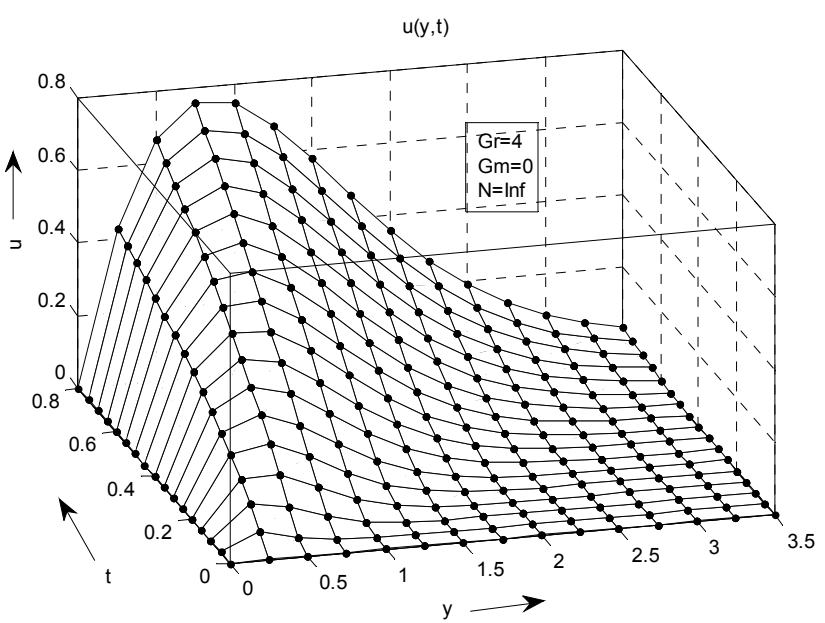

Fig.4. Variation of velocity with $y$ and $t$ in the case of isothermal plate $(r=1)$ in the absence of both mass transfer and radiation.

A comparison of Fig. 1 with Figs 2, 3 and 4 reveals that there is a rise in velocity due to the presence of radiation and/or diffusing species as compared to the cases of no radiation and/or no diffusing species.

In Figs 7 and 8, two dimensional graphs have been drawn to depict the variation of velocity $u$ with similarity variable $\eta$ for different values of the radiation parameter $N$, in the presence and absence of mass transfer, respectively, at $t=0.4$. It may be observed from these figures that for an increase in parameter $N$, there is a fall in the velocity whereas for an increase in $\mathrm{Gr}$ or Gm, there is a rise in the velocity. Further, the absence of radiation heat transfer and/or mass transfer causes the velocity to decrease which is in conformity with the observation drawn from 3D Figs 1-4.

\section{Case (ii) $r=2$ (Plate temperature and concentration vary linearly with time):}

This is the case of an initial unsteady flow when the temperature and concentration at the plate are both linear functions of time. In this case, Fig.5 displays a 3D graph for velocity distribution $u(y, t)$ for $\mathrm{Gr}=4$, 
$\mathrm{Gm}=4, N=3$ and Fig.9 displays a 2D graph for velocity $u$ against $\eta$. Comparing Figs 1 and 5, it may be observed that there is a fall in the velocity when the plate temperature and concentration vary linearly with time for a fixed time $t<1$. The effects of other parameters are same as those observed in case (i).

\section{Case (iii) $r=3$ (Plate temperature and concentration vary non-linearly with time):}

This is the case of an initial unsteady flow when the temperature and concentration at the plate are both quadratic functions of time. In this case, Fig.6 displays a 3D graph for velocity distribution $u(y, t)$ for $\mathrm{Gr}=4, \mathrm{Gm}=4, N=3$ and Fig. 10 displays a 2D graph for velocity $u$ against $\eta$. Comparing Fig. 6 with Figs 1 and 5 , it may be observed that there is a further fall in the velocity when the plate temperature and concentration vary non-linearly with time for a fixed time $t<1$, as compared to cases (i) and (ii).



Fig.5. Variation of velocity with $y$ and $t$ in the case of plate temperature varying linearly with time $(r=2)$.



Fig.7. Variation of velocity $(u)$ with similarity variable $(\eta)$ in the case of isothermal plate $(r=1)$.

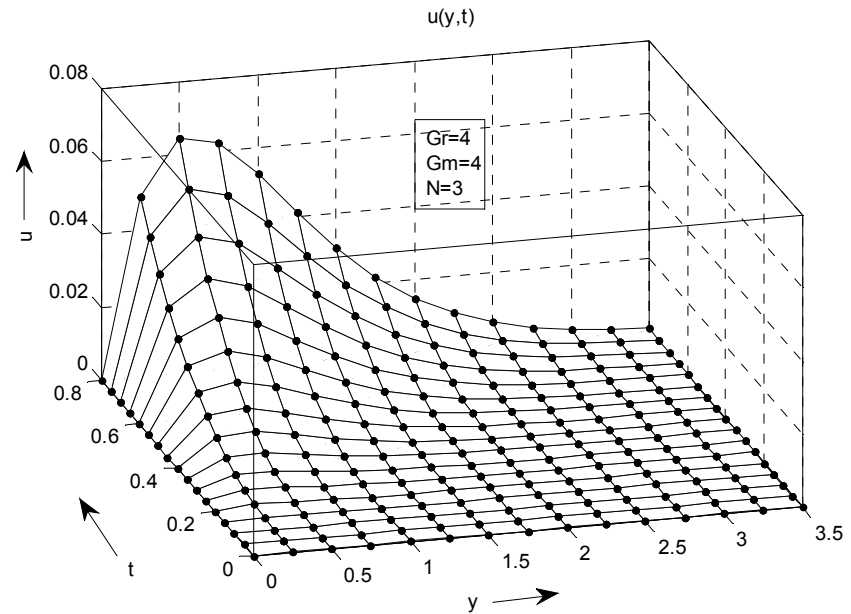

Fig.6. Variation of velocity with $y$ and $t$ in the case of plate temperature varying non-linearly with time $(r=3)$.

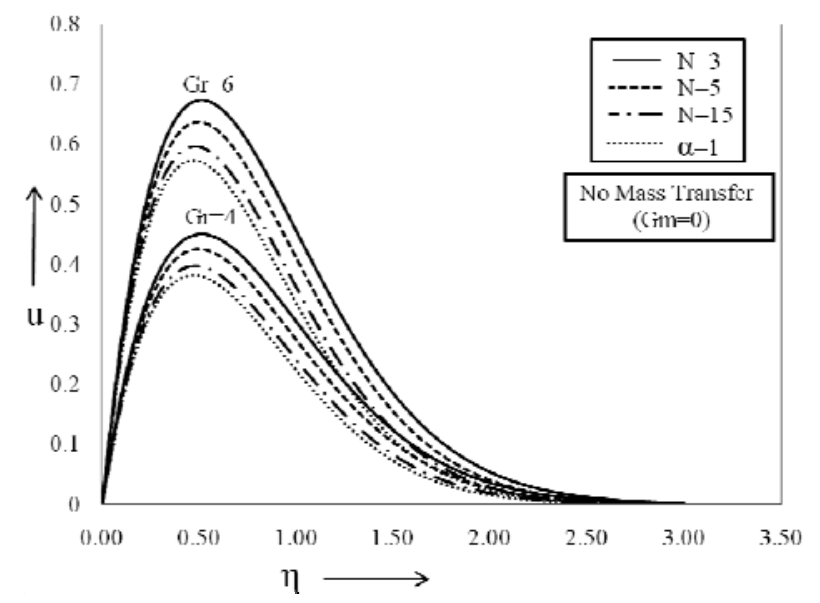

Fig.8. Velocity variation of velocity $(u)$ with similarity variable $(\eta)$ in the case of isothermal plate $(r=1)$ in the absence of mass transfer. 


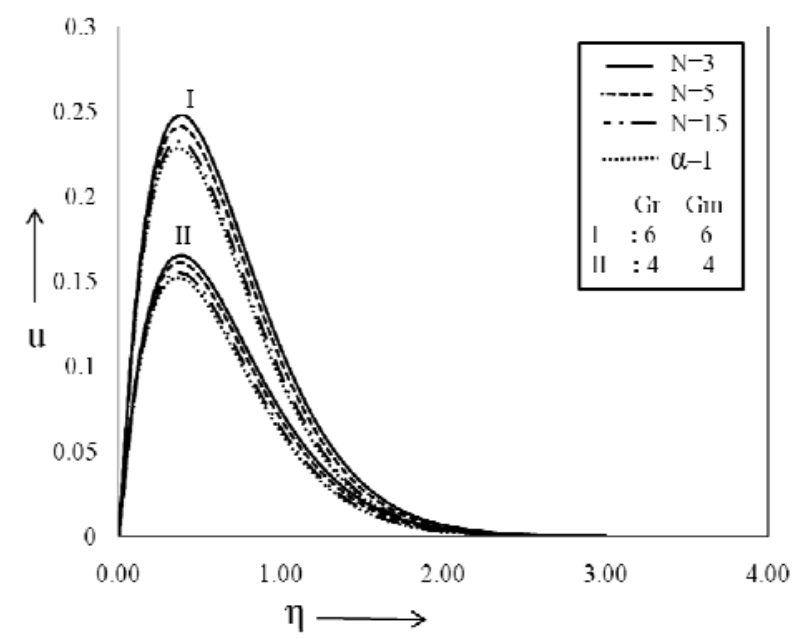

Fig.9. Variation of velocity $(u)$ with similarity variable $(\eta)$ in the case of plate temperature varying linearly with time $(r=2)$.

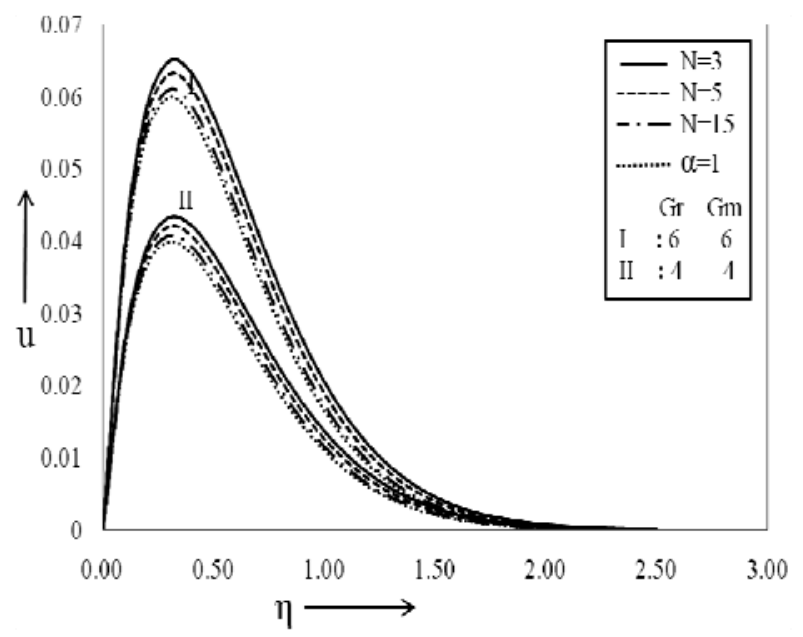

Fig.10. Variation of velocity $(u)$ with similarity variable $(\eta)$ in the case of plate temperature varying non-linearly with time $(r=3)$

Finally, the temperature function $\theta$ and concentration function $\mathrm{C}$ have been plotted against the similarity variable $\eta$ for different values of $r$ and $N$ in Figs 11 and 12, respectively.

It is noted from Fig.11 that for all values of $r$ there is a rise in temperature due to the presence of thermal radiation as compared to the case of no radiation. Further, the temperature decreases with increasing values of $N$. As $N \rightarrow \infty$ (no radiation case), the limiting temperature profile is also shown in Fig.11, which is the minimum temperature profile as compared to the cases of radiation $(N=3,5,15)$. We also observe that the temperature decreases as $r$ increases.

In Fig.12, species concentration profiles are shown against $\eta$, for various values of $r$. We observe that the species concentration decreases as $r$ increases.



Fig.11. Temperature profile for $\operatorname{Pr}=0.71$ and various values of $N$.



Fig.12. Species concentration profile for $\mathrm{Sc}=0.6$ and various values of $r$. 
We have also calculated numerical values of the skin-friction $C_{f}$ that are listed in Tabs 1-3. We observe from Tab. 1 that a decrease in the radiation parameter $N$ or an increase in the mass transfer parameter $\mathrm{Gm}$ or Grashof numbers Gr or time $t$, leads to an increase in the skin-friction. We also observe from Tabs 1 , 2 and 3 that the skin-friction decreases as $r$ increases.

In Tab.4, numerical values of the skin-friction $C_{f}$, in the absence of radiation, are listed. We compare these values of $C_{f}$ with those in Tabs 1-3 and conclude that the value of the skin-friction in the presence of radiation is greater than that in the non-radiation case, other parameters being fixed.

Hence, the presence of radiation causes a rise in the skin-friction when other parameters are fixed. Also, we observe that the presence of species concentration causes a rise in the skin-friction when other parameters are fixed.

In Tab.3, numerical values of the skin-friction $C_{f}$, in the absence of radiation, are listed. We compare these values of $C_{f}$ with those in Tabs 1-2 and conclude that the value of skin-friction is less than that in the non-radiation case. Hence, the presence of radiation causes a fall in the skin-friction at various values of $t$ and Gr. Also observe that the presence of species concentration causes a rise in the skin-friction at various values of $t$ and $\mathrm{Gr}$.

Table 1. Numerical values of skin-friction of isothermal plates for $\operatorname{Pr}=0.71, \mathrm{Sc}=0.6$.

\begin{tabular}{|c|c|c|c|c|}
\hline Gr & $\mathbf{G m}$ & $\mathbf{N}$ & $\mathbf{T}$ & $\mathbf{C . F .}$ \\
\hline 2 & 2 & 3 & 0.2 & 2.324035 \\
\hline 4 & 4 & 3 & 0.2 & 4.64807 \\
\hline 6 & 6 & 3 & 0.2 & 6.972105 \\
\hline 2 & 2 & 5 & 0.2 & 2.291746 \\
\hline 4 & 4 & 5 & 0.2 & 4.583491 \\
\hline 6 & 6 & 5 & 0.2 & 6.875237 \\
\hline 2 & 2 & 15 & 0.2 & 2.25419 \\
\hline 4 & 4 & 15 & 0.2 & 4.50838 \\
\hline 6 & 6 & 15 & 0.2 & 6.76257 \\
\hline 2 & 2 & 3 & 0.4 & 3.286682 \\
\hline 4 & 4 & 3 & 0.4 & 6.573363 \\
\hline 6 & 6 & 3 & 0.4 & 9.860045 \\
\hline 2 & 2 & 5 & 0.4 & 3.241018 \\
\hline 4 & 4 & 5 & 0.4 & 6.482036 \\
\hline 6 & 6 & 5 & 0.4 & 9.723053 \\
\hline 2 & 2 & 15 & 0.4 & 3.187906 \\
\hline 4 & 4 & 15 & 0.4 & 6.375812 \\
\hline 6 & 6 & 15 & 0.4 & 9.563718 \\
\hline 2 & 0 & 3 & 0.4 & 1.678092 \\
\hline 4 & 0 & 3 & 0.4 & 3.356183 \\
\hline 6 & 0 & 3 & 0.4 & 5.034276 \\
\hline 2 & 0 & 5 & 0.4 & 1.632428 \\
\hline 4 & 0 & 5 & 0.4 & 3.264855 \\
\hline 6 & 0 & 5 & 0.4 & 4.897283 \\
\hline 2 & 0 & 15 & 0.4 & 1.579316 \\
\hline 4 & 0 & 15 & 0.4 & 3.158632 \\
\hline 6 & 0 & 15 & 0.4 & 4.737948 \\
\hline & & & & \\
\hline
\end{tabular}

Table 2. Numerical values of skin-friction in the case of plate temp varying linearly for $\mathrm{Pr}=0.71, \mathrm{Sc}=0.6$.

\begin{tabular}{|c|c|c|c|c|}
\hline $\mathbf{G r}$ & $\mathbf{G m}$ & $\mathbf{N}$ & $\mathbf{T}$ & $\mathbf{C . F .}$ \\
\hline 2 & 2 & 3 & 0.2 & 0.3098713 \\
\hline 4 & 4 & 3 & 0.2 & 0.6197426 \\
\hline 6 & 6 & 3 & 0.2 & 0.929614 \\
\hline 2 & 2 & 5 & 0.2 & 0.3055661 \\
\hline 4 & 4 & 5 & 0.2 & 0.6111322 \\
\hline 6 & 6 & 5 & 0.2 & 0.9166982 \\
\hline 2 & 2 & 15 & 0.2 & 0.3005587 \\
\hline 4 & 4 & 15 & 0.2 & 0.6011174 \\
\hline 6 & 6 & 15 & 0.2 & 0.901676 \\
\hline 2 & 2 & 3 & 0.4 & 0.8764485 \\
\hline 4 & 4 & 3 & 0.4 & 1.752897 \\
\hline 6 & 6 & 3 & 0.4 & 2.629345 \\
\hline 2 & 2 & 5 & 0.4 & 0.8642714 \\
\hline 4 & 4 & 5 & 0.4 & 1.728543 \\
\hline 6 & 6 & 5 & 0.4 & 2.592814 \\
\hline 2 & 2 & 15 & 0.4 & 0.8501083 \\
\hline 4 & 4 & 15 & 0.4 & 1.700217 \\
\hline 6 & 6 & 15 & 0.4 & 2.550325 \\
\hline 2 & 0 & 3 & 0.4 & 0.4474911 \\
\hline 4 & 0 & 3 & 0.4 & 0.8949822 \\
\hline 6 & 0 & 3 & 0.4 & 1.342474 \\
\hline 2 & 0 & 5 & 0.4 & 0.4353141 \\
\hline 4 & 0 & 5 & 0.4 & 0.8706281 \\
\hline 6 & 0 & 5 & 0.4 & 1.305942 \\
\hline 2 & 0 & 15 & 0.4 & 0.421151 \\
\hline 4 & 0 & 15 & 0.4 & 0.8423019 \\
\hline 6 & 0 & 15 & 0.4 & 1.263453 \\
\hline & & & & \\
\hline
\end{tabular}


Table 3. Numerical values of skin-friction for $\operatorname{Pr}=0.71, \mathrm{Sc}=0.6, t=0.4$ and $\alpha=1$ (no radiation case).

\begin{tabular}{|c|c|c|c|}
\hline $\boldsymbol{r}$ & $\mathbf{G r}$ & $\mathbf{G m}$ & $\mathbf{C . F}$. \\
\hline 1 & 2 & 2 & 3.157801 \\
\hline 1 & 4 & 4 & 6.315601 \\
\hline 1 & 6 & 6 & 9.473401 \\
\hline 1 & 2 & 0 & 1.549211 \\
\hline 1 & 4 & 0 & 3.098421 \\
\hline 1 & 6 & 0 & 4.647632 \\
\hline 2 & 2 & 2 & 0.8420802 \\
\hline 2 & 4 & 4 & 1.68416 \\
\hline 2 & 6 & 6 & 2.526241 \\
\hline 2 & 2 & 0 & 0.4131228 \\
\hline 2 & 4 & 0 & 0.8262456 \\
\hline 2 & 6 & 0 & 1.239368 \\
\hline
\end{tabular}

Table 4. Numerical values of skin-friction for $\operatorname{Pr}=0.71, \mathrm{Sc}=0.6, t=0.4$ and $\alpha=1$ (no radiation case).

\begin{tabular}{|c|c|c|c|}
\hline $\mathbf{r}$ & Gr & Gm & C.F. \\
\hline 1 & 2 & 2 & 3.157801 \\
\hline 1 & 4 & 4 & 6.315601 \\
\hline 1 & 6 & 6 & 9.473401 \\
\hline 1 & 2 & 0 & 1.549211 \\
\hline 1 & 4 & 0 & 3.098421 \\
\hline 1 & 6 & 0 & 4.647632 \\
\hline 2 & 2 & 2 & 0.8420802 \\
\hline 2 & 4 & 4 & 1.68416 \\
\hline 2 & 6 & 6 & 2.526241 \\
\hline 2 & 2 & 0 & 0.4131228 \\
\hline 2 & 4 & 0 & 0.8262456 \\
\hline 2 & 6 & 0 & 1.239368 \\
\hline 3 & 2 & 2 & 0.2694657 \\
\hline 3 & 4 & 4 & 0.5389313 \\
\hline 3 & 6 & 6 & 0.8083969 \\
\hline 3 & 2 & 0 & 0.1321993 \\
\hline 3 & 4 & 0 & 0.2643986 \\
\hline 3 & 6 & 0 & 0.3965979 \\
\hline
\end{tabular}

\section{Nomenclature}

$C$ - species concentration

$C_{f}-$ skin friction coefficient

$C_{p}$ - specific heat at constant pressure

$C_{\infty}$ - concentration at infinity

$D$ - chemical molecular diffusivity 
Gm - Grashof number for mass transfer

Gr - Grashof number

$g$ - acceleration due to gravity

$i^{n} \operatorname{erf}_{\mathrm{c}}(z)$ - repeated integral of complementary error function

$N$ - radiation parameter

Pr - Prandtl number

Sc - Schmidt number

$T$ - temperature of the fluid

$T_{\infty} \quad$ - temperature of the fluid far away from the plate

$T_{w} \quad$ - plate temperature

$t$ - time

$u$ - velocity component

$u_{0}$ - constant with dimension of velocity

$q_{r}$ - radiative heat flux

$v$ - kinematic viscosity

$\beta-$ coefficient of volume expansion

$\beta^{*}-$ volumetric coefficient of expansion with species concentration

$\theta$ - dimensionless temperature

$\kappa-$ thermal conductivity of the fluid

$\kappa^{*}-$ mean absorption coefficient

$\rho-$ density

$\sigma \quad$ - Stefan-Boltzmann constant

\section{References}

[1] Abramowitz M. and Stegun I.A. (1972): Handbook of Mathematical Functions with Formulas, Graphs, and Mathematical Tables. - New York: Dover Publications.

[2] Bansal J.L. (1994): Magnetofluiddynamics of Viscous Fluids. - Jaipur: Jaipur Publishing House.

[3] Brewster M.Q. (1992): Thermal Radiative Transfer and Properties. - John Wiley and Sons. Ind.

[4] Chaudhary R.C. and Jain P. (2006): Unsteady free convection boundary layer flow past an impulsively started vertical surface with Newtonian heating. - Romanian Journal of Physics, vol.51, pp.911-925.

[5] Chandrakala P. (2010): Radiation effects on flow past an impulsively started vertical oscillating plate with uniform heat flux. - International Journal of Dynamics of Fluids, vol.6, pp.209-215.

[6] Chandrakala P. and Bhaskar P.N. (2012): Radiation effects on oscillating vertical plate with uniform heat flux and mass diffusion. - International Journal of Fluids Engineering, vol.4, pp.1-11.

[7] Chandran P., Sacheti N.C. and Singh A.K. (2005): Natural convection near a vertical plate with ramped wall temperature. - Heat and Mass Transfer, vol.41, pp.459-464.

[8] Chien-Hsin-Chen (2004): Combined heat and mass transfer in MHD free convection from a vertical surface with Ohmic heating and viscous dissipation. - Int. J. Engineering Science, vol.42, No.7, pp.699-713.

[9] Deka R.K. and Das S.K. (2011): Radiation effects on free convection flow near a vertical plate with ramped wall temperature. - Engineering, vol.3, pp.1197-1206.

[10] Korycki R. (2006): Sensitivity analysis and shape optimization for transient heat conduction with radiation. International Journal of Heat and Mass Transfer, vol.49 No.13-14 pp.2033-2043.

[11] Mebine P. and Adigio E.M. (2009): Unsteady free convection flow with thermal radiation past a vertical porous plate with Newtonian heating. - Turkish Journal of Physics, vol.33, No.2, pp.109-119. 
[12] Muthucumaraswamy R. (2004): Natural convection on flow past an impulsively started vertical platewith variable surface heat flux. - Far East Journal of Applied Mathematics, vol.14, no.1, pp.99-119.

[13] Muthucumaraswamy R., Chandrakala P. and Raj S.A. (2006): Radiative heat and mass transfer effects on moving isothermal vertical plate in the presence of chemical reaction. - International Journal of Applied Mechanics and Engineering, vol.11, No.3, pp.639-646.

[14] Muthukumarswamy R. and Senthil G.K. (2004): Heat and Mass transfer effect on moving vertical plate in the presence of thermal radiation. - Theoret. Appl. Mech. vol.31, pp.35-46.

[15] Muthucumaraswamy R. and Vijayalakshmi A. (2008): Effects of heat and mass transfer on flow past an oscillating vertical plate with variable temperature. - International Journal of Applied Mathematics and Mechanics, vol.4, No.1, pp.59-65.

[16] Narahari M. and Ishak A. (2011): Radiation effects on free convection flow near a moving vertical plate with newtonian heating. - Journal of Applied Sciences, vol.11, No.7, pp.1096-1104.

[17] Narahari M. and Yunus Nayan M. (2011): Free convection flow past an impulsively started infinite vertical plate with Newtonian heating in the presence of thermal radiation and mass diffusion. - Turkish Journal of Engineering and Environmental Sciences, vol.35, No.3, pp.187-198.

[18] Pathak G., Maheshwari C.H. and Gupta S.P. (2006): Effects of radiation on unsteady free convection flow bounded by an oscillating plate with variable temperature. - International Journal of Applied Mechanics and Engineering, vol.11, pp.371-382.

[19] Prasad V.R., Reddy N.B. and Muthucumaraswamy R. (2007): Radiation and mass transfer effects on twodimensional flow past an impulsively started infinite vertical plate. - International Journal of Thermal Sciences, vol.46, No.12, pp.1251-1258.

[20] Rajesh V. (2012): Effects of mass transfer on flow past an impulsively started infinite vertical plate with Newtonian heating and chemical reaction. - Journal of Engineering Physics and Thermophysics, vol.85, No.1, pp.221-228.

[21] Soundalgekar V.M. (1977): Free convection effects on the Stokes problem for an infinite vertical plate. - J. Heat Transfer (Trans. ASME), 99C: pp.499-501.

[22] Soundalgekar V.M. and Patil M.R. (1980): Stokes problem for infinite vertical plate with constant heat flux. Astrophys. Space Sci., vol.70, pp.179-182.

[23] Tak S.S. and Parihar Renu (2005): Radiation and mass transfer effects on flow past an impulsively started infinite vertical plate. - Bulletin of pure and Applied Science, vol.24E, No.2 pp.221-231.

[24] Vighnesam N.V. and Soundalgekar V.M. (2001): Heat Transfer in MHD stagnation point flow with variable temperature. - Bull. Cal. Math. Soc., vol.93, No.3, pp.229-232.

Received: July 14, 2016

Revised: September 20, 2017 